\title{
Attenuation of MUC4 potentiates the anticancer activity of auranofin via regulation of the Her2/Akt/FOXO3 pathway in ovarian cancer cells
}

\author{
JUN SANG BAE ${ }^{1 *}$, JONGSUNG LEE $^{2 *}$, YOONKOOK PARK ${ }^{3}$, KYUNGMOON PARK $^{3}$, JUNG RYUL KIM $^{4}$, \\ DONG HYU $\mathrm{CHO}^{5}, \mathrm{KYU}^{\mathrm{Y} U N} \mathrm{JANG}^{1}$ and SEE-HYOUNG PARK ${ }^{3}$
}

\author{
${ }^{1}$ Department of Pathology, Chonbuk National University Medical School, Research Institute of Clinical Medicine of \\ Chonbuk National University-Biomedical Research Institute of Chonbuk National University Hospital and Research Institute \\ for Endocrine Sciences, Jeonju; ${ }^{2}$ Department of Genetic Engineering, Sungkyunkwan University, Suwon; \\ ${ }^{3}$ Department of Bio and Chemical Engineering, Hongik University, Sejong; ${ }^{4}$ Department of Orthopaedic Surgery, \\ Chonbuk National University Medical School, Research Institute of Clinical Medicine of \\ Chonbuk National University-Biomedical Research Institute of Chonbuk National University Hospital \\ and Research Institute for Endocrine Sciences; ${ }^{5}$ Department of Obstetrics and Gynecology, \\ Chonbuk National University Medical School, Research Institute of Clinical Medicine of Chonbuk National University- \\ Biomedical Research Institute of Chonbuk National University Hospital, Jeonju, Republic of Korea
}

Received February 24, 2017; Accepted July 17, 2017

DOI: $10.3892 /$ or.2017.5853

\begin{abstract}
Previously, we reported that auranofin induces apoptosis in SKOV3 cells via regulation of the IKK $\beta / F O X O 3$ pathway. In the present study, we reveal that the anticancer activity of auranofin in SKOV3 cells could be enhanced by the attenuation of MUC4 through the regulation of the Her2/Akt/FOXO3 pathway. Compared to the control-siRNA, siRNA transfection against MUC4 into SKOV3 cells accelerated the protein degradation of Her2. Under the same conditions, the expression level of phosphorylated Akt was also downregulated leading to an increase of FOXO3 in the nucleus. Notably, auranofin treatment in SKOV3 cells also resulted in the downregulation of the expression levels of both Her2 and phosphorylated Akt. Thus, Her2 was identified as the common molecular target protein by siRNA transfection against MUC4. Western blot analysis of total and nuclear
\end{abstract}

Correspondence to: Professor Kyu Yun Jang, Department of Pathology, Chonbuk National University Medical School, Research Institute of Clinical Medicine of Chonbuk National UniversityBiomedical Research Institute of Chonbuk National University Hospital and Research Institute for Endocrine Sciences, Jeonju, Republic of Korea

E-mail: kyjang@jbnu.ac.kr

Professor See-Hyoung Park, Department of Bio and Chemical Engineering, Hongik University, Sejong, Republic of Korea

E-mail: shpark74@hongik.ac.kr

*Contributed equally

Key words: MUC4, auranofin, Her2, Akt, FOXO3, ovarian, cancer fraction lysates from SKOV3 cells revealed that attenuation of MUC4 combined with auranofin treatment in SKOV3 cells synergistically activated FOXO3 translocation from the cytoplasm to the nucleus through the regulation of the Her2/Akt/FOXO3 pathway. Attenuation of MUC4 by siRNA transfection potentiated the antitumor effect of auranofin which was examined by performing in vitro assays such as WST-1, cell counting, colony formation, TUNEL and Annexin V staining. In addition, western blot analysis of the apoptosis-related proteins such as PARP1, caspase-3, Bim extra large (EL), Bax and $\mathrm{Bcl} 2$ revealed that the attenuation of MUC4 by siRNA transfection potentiates the pro-apoptotic activity of auranofin in SKOV3 cells. Collectively, auranofin could regulate the Her2/Akt/FOXO3 signaling pathway in SKOV3 cells and be used as a potential antitumor agent considering the expression of MUC4 in ovarian cancer patients.

\section{Introduction}

Recently, was reported that mucin expression and its glycosylation patterns are altered abnormally in inflammatory, premalignant and malignant conditions (1-6). Mucins are known as glycoproteins with $O$ - and $N$-oligosaccharides and contain multiple tandem repeats of 10-80 amino acid residues (5). There are two categories of mucins; one type is membrane-bound, and the other is secreted/gel forming. It is well known that MUC1 and MUC4 are the most characterized transmembrane mucins which are significant in cellular physiology. The structure and biochemical composition of these mucins helps them to offer lubrication and hydration to cell surfaces as well as provide protection from pathogens and degradative enzymes (7). Not only the expression of MUC1 and MUC4, but also the glycosylation pattern is subject to vary 
and it has been demonstrated in studies of several malignant epithelial tumors, such as pancreatic, lung, colon, breast and prostate cancer $(3,5,8,9)$.

Studies on MUC1 and MUC4 revealed that they play pivotal roles in cellular signaling, tumor immune surveillance, tumor growth, metastasis, tumor-stromal cell interactions and chemotherapy resistance $(3,7,8,10)$. There are a few reagents which have high specificity (e.g. monoclonal antibodies) which can even recognize modified glycoforms available, suggesting that mucins can be useful targets enabling the detection of malignant epithelial tumors at an early stage $(11,12)$. Studies in malignant ovarian neoplasms provide casual evidence that the expression as well as the localization pattern of MUC1 are modified during their progression, nevertheless, in the case of MUC4, more information is required (13). In light of the fact that MUC1 and MUC4 participate in the lubrication of cell surfaces and provide protection, it is critical to observe their functions as well as the variation of their expression while malignant ovarian tumors develop and progress.

Her2 is a receptor tyrosine kinase that is a member of the transmembrane epidermal growth factor type II receptor family and is also known as erbB-2/CD340. Her2 overexpression has been recognized as a stable molecular abnormality, driven in several of the most common solid tumors including prostate, cervical, ovarian, breast, lung, endometrial and colon cancer (14-17). It has also been proposed that there is a significant connection between Her2 overexpression and a poor prognosis in lymph node-positive/negative patients with breast cancer. Furthermore, it can be a strong marker for the therapy and diagnosis of other solid tumors, e.g. multiple gynecological cancers (18). Recently, MUC4 was revealed to be involved in the development of ovarian cancer through the stabilization and activation of Her2 $(19,20)$. Thus, it may be worth studying the detailed signaling pathways related to the MUC4/Her2 pathway that could be targeted as novel therapeutic options to treat ovarian cancer.

Previously, we identified auranofin, a rheumatoid arthritis therapeutic agent approved by the Food and Drug Administration (FDA), as a FOXO3 activator and revealed that auranofin induces apoptosis in SKOV3 cells via the regulation of the IKK $\beta / \mathrm{FOXO} 3$ pathway (21). In the present study, we demonstrated that auranofin regulates Her2 protein expression and that the anticancer activity of auranofin in SKOV3 cells could be enhanced by the attenuation of MUC4 through the regulation of the Her2/Akt/FOXO3 pathway. The present study may be helpful in the selection of a potential antitumor agent considering the expression of MUC4 in ovarian cancer patients.

\section{Materials and methods}

Cell lines. SKOV3, OVACAR5, MDA-MB-231 and MDA-MB361 cells [from the American Tissue Culture Collection (ATCC) Manassas, VA, USA] were maintained in Dulbecco's modified Eagle's medium (DMEM) supplemented with $10 \%$ fetal bovine serum, and $1 \%$ streptomycin/penicillin at $37^{\circ} \mathrm{C}$ in a humidified incubator containing $5 \% \mathrm{CO}_{2}$ in air.

Antibodies and chemical reagents. Mouse anti- $\beta$-actin antibody, dimethyl sulfoxide (DMSO), glycerol, glycine, sodium chloride, Trizma base and Tween-20 were purchased from Sigma-Aldrich (St. Louis, MO, USA). Mouse anti-PARP1, rabbit anti-FOXO3, mouse anti-LaminA/C antibodies and auranofin were purchased from Santa Cruz Biotechnology (Santa Cruz, CA, USA). Rabbit anti-Her3, rabbit anti-Her2, rabbit anti-phospho-FOXO3, rabbit anti-phospho-Akt, rabbit anti-Akt, rabbit anti-caspase-3, rabbit anti-Bax, rabbit antiBim and rabbit anti-Bcl2 antibodies were obtained from Cell Signaling Technology, Inc. (Danvers, MA, USA). The rabbit anti-MUC4 antibody was purchased from Abcam (Cambridge, MA). Goat anti-mouse and goat anti-rabbit horseradish peroxidase-conjugated $\mathrm{IgG}$ were obtained from Jackson ImmunoResearch (West Grove, PA, USA). ECL Western Blotting Detection Reagents were obtained from GenDEPOT (Barker, TX, USA).

WST-1 cell viability assay. A $200 \mu \mathrm{l}$ aliquot of SKOV3 cells ( $1 \times 10^{3}$ cells in media) was added to each well of a $96-$ well plate and incubated for $18 \mathrm{~h}$ at $37^{\circ} \mathrm{C}$ in a humidified incubator containing $5 \% \mathrm{CO}_{2}$ in air. After incubation, control or MUC4-siRNA (Santa Cruz Biotechnology) was transfected followed by the addition of auranofin ( 0 or $25 \mathrm{nM})$ into each well for $48 \mathrm{~h}$. After incubation, a $20 \mu \mathrm{l}$ WST-1 solution was added to each well and the incubation continued for $2 \mathrm{~h}$. The visible absorbance at $560 \mathrm{~nm}$ of each well was quantified using a microplate reader.

Cell counting assay. SKOV3 cells $\left(1 \times 10^{4}\right)$ were seeded in $6-\mathrm{cm}$ dishes and incubated at $37^{\circ} \mathrm{C}$ in a humidified incubator containing $5 \% \mathrm{CO}_{2}$ in air for $18 \mathrm{~h}$. After incubation, control or MUC4-siRNA was transfected followed by the addition of auranofin ( 0 or $25 \mathrm{nM}$ ) into each dish for $0,24,48$ and $72 \mathrm{~h}$. The number of cells were assessed daily using a hemocytometer.

Colony formation assay. SKOV3 cells $\left(5 \times 10^{2}\right)$ were seeded in 6-cm dishes and incubated at $37^{\circ} \mathrm{C}$ in a humidified incubator containing $5 \% \mathrm{CO}_{2}$ in air for $18 \mathrm{~h}$. After incubation, control or MUC4-siRNA was transfected followed by the addition of auranofin ( 0 or $25 \mathrm{nM}$ ) into each dish for 7 days. Subsequently, the colonies were washed twice with phosphate-buffered saline (PBS), fixed with $3.7 \%$ paraformaldehyde, and stained with $1 \%$ crystal violet solution in distilled water.

Western blot analysis. Cells were washed with PBS and lysed in lysis buffer (50 mM Tris- $\mathrm{HCl}, 150 \mathrm{mM} \mathrm{NaCl}, 2 \mathrm{mM}$ EDTA, $1 \%$ Triton $\mathrm{X}-100,0.1 \%$ SDS, $\mathrm{pH}$ 8.0) with protease and phosphatase inhibitors. Cell lysates were centrifuged $(10,000 \mathrm{x} \mathrm{g}$, $4^{\circ} \mathrm{C}, 10 \mathrm{~min}$ ) and the supernatants were separated on 6 or $10 \%$ SDS-PAGE and blotted onto nitrocellulose membranes (Bio-Rad Laboratories, Hercules, CA, USA). The membranes were blocked in 3\% non-fat dry milk for $1 \mathrm{~h}$ at room temperature, and probed with appropriate antibodies. The membranes were then probed with HRP-tagged anti-mouse or anti-rabbit IgG antibodies diluted 1:5,000-1:15,000 in 3\% non-fat dry milk for $1 \mathrm{~h}$ at room temperature. Chemiluminescence was detected using enhanced ECL.

Cytoplasmic and nuclear protein fractionation. Cells from each condition were trypsinized, centrifuged, washed, re-suspended in a cytoplasmic fractional buffer $(10 \mathrm{mM}$ 


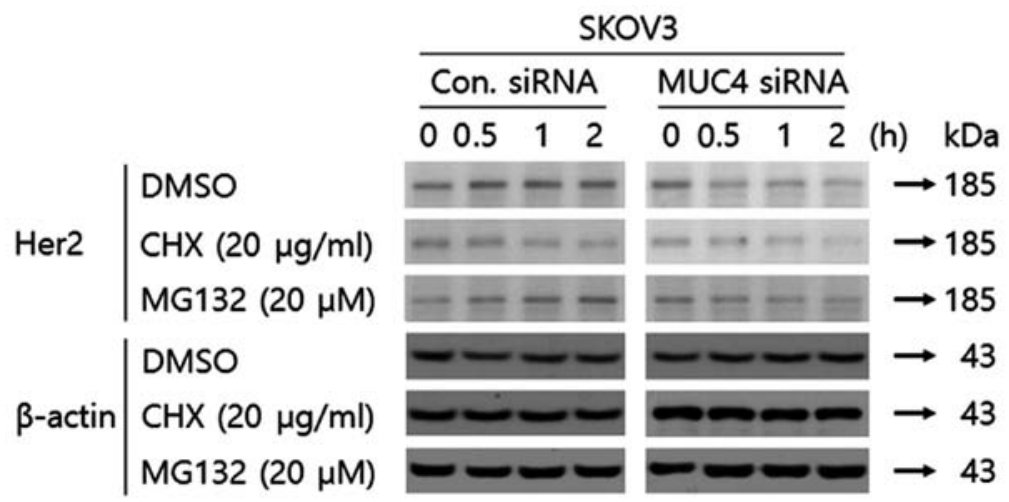

Figure 1. The protein expression level of Her2 is decreased faster in cells transfected with MUC4-siRNA than in cells transfected with control-siRNA. SKOV3 cells $\left(1 \times 10^{5}\right)$ were seeded in $6-\mathrm{cm}$ dishes and incubated at $37^{\circ} \mathrm{C}$ in a humidified incubator containing $5 \% \mathrm{CO}_{2}$ in air for $18 \mathrm{~h}$. After incubation, the control or MUC4-siRNA was transfected. Then, the cells were treated with DMSO (control vehicle), cycloheximide (CHX; $20 \mu \mathrm{g} / \mathrm{ml}$ ), or MG-132 (20 $\mu \mathrm{M})$ for the indicated times. Cell lysates were immune-blotted with anti-Her 2 antibodies. $\beta$-actin was used as the loading control.

HEPES, pH 8.0, $50 \mathrm{mM} \mathrm{NaCl}, 500 \mathrm{mM}$ sucrose, $1 \mathrm{mM}$ EDTA, $0.5 \mathrm{mM}$ spermidine, $0.15 \mathrm{mM}$ spermine, $0.2 \%$ Triton $\mathrm{X}-100$, $1 \mathrm{mM}$ DTT, $2 \mu \mathrm{M}$ phenylmethylsulfonyl fluoride (PMSF) and $0.15 \mathrm{U} / \mathrm{ml}$ aprotinin, and incubated at $4^{\circ} \mathrm{C}$ for $30 \mathrm{~min}$ on a rotator. After centrifuging the cell suspension at 10,000 rpm for $30 \mathrm{~min}$ at $4^{\circ} \mathrm{C}$, the supernatant was collected for cytoplasmic fractioning. The pellet was washed with washing buffer (10 mM HEPES pH 8.0, $50 \mathrm{mM} \mathrm{NaCl,} \mathrm{25 \%} \mathrm{glycerol,}$ $0.1 \mathrm{mM}$ EDTA, $0.5 \mathrm{mM}$ spermidine and $0.15 \mathrm{mM}$ spermine) twice. The remaining pellet was re-suspended with a nuclear fractional buffer (10 mM HEPES pH 8, $350 \mathrm{mM} \mathrm{NaCl}, 25 \%$ glycerol, $0.1 \mathrm{mM}$ EDTA, $0.5 \mathrm{mM}$ spermidine and $0.15 \mathrm{mM}$ spermine) and incubated at $4^{\circ} \mathrm{C}$ for $30 \mathrm{~min}$ on a rotator. After centrifuging the cell suspension at $13,000 \mathrm{rpm}$ for $30 \mathrm{~min}$ at $4^{\circ} \mathrm{C}$, the supernatant was collected for nuclear fractioning. Protein in each fraction was quantified using the Bradford protein determination reagent (Bio-Rad Laboratories) and BSA as a standard.

TUNEL assay. SKOV 3 cells $\left(1 \times 10^{4}\right)$ were seeded in 6-cm dishes and incubated at $37^{\circ} \mathrm{C}$ in a humidified incubator containing $5 \% \mathrm{CO}_{2}$ in air for $18 \mathrm{~h}$. After incubation, control or MUC4siRNA was transfected followed by the addition of auranofin ( 0 or $25 \mathrm{nM}$ ) into each dish for $2 \mathrm{~h}$. Then, the cells were fixed with $4 \%$ paraformaldehyde solution and permeabilized with Triton X-100 (0.2\%). For the TUNEL assay, cellular apoptosis was determined by enzymatic labeling of DNA strand breaks with a TUNEL assay kit (the DeadEnd Fluorometric TUNEL System; Promega, Madison, WI, USA) according to the manufacturer's instructions. Nuclei were stained with 4',6-diamidine-2'-phenylindole dihydrochloride (DAPI).

Annexin V staining analysis. The percentage of cells that underwent apoptosis was determined using the FITC Annexin V apoptosis detection kit I (BD Pharmingen, San Diego, CA, USA) with propiodium iodide (PI) according to the manufacturer's instructions. Briefly, SKOV3 cells $\left(1 \times 10^{4}\right)$ were seeded in 6-cm dishes and incubated at $37^{\circ} \mathrm{C}$ in a humidified incubator containing $5 \% \mathrm{CO}_{2}$ in air for $18 \mathrm{~h}$. After incubation, control or MUC4-siRNA was transfected followed by the addition of auranofin ( 0 or $25 \mathrm{nM}$ ) into each dish for $2 \mathrm{~h}$. Subsequently, the cells were washed in PBS, trypsinized and resuspended in binding buffer. Then, the cells were aliquoted into $5 \mathrm{ml}$ culture tubes $\left(1 \times 10^{5}\right.$ cells/tube $)$, and incubated in binding buffer containing $5 \mu \mathrm{l}$ of FITC Annexin V, and $5 \mu \mathrm{l}$ of PI for $15 \mathrm{~min}$ at $25^{\circ} \mathrm{C}$ in the dark. The cells were then analyzed using FACSCalibur (BD Biosciences, Franklin Lakes, NJ, USA) and the data were analyzed by FlowJo (De Novo Software, Glendale, CA, USA). Ten thousand events were collected in each run.

Statistical analysis. The results are expressed as arithmetic mean \pm SEM (the standard error of the mean). To compare the statistical meaning between the groups, two-sided unpaired Student's t-tests were used. All experiments were repeated 3 times and the representative data are shown. Statistical analyses were performed using SPSS software (version 19.0; SPSS, Inc., Chicago, IL, USA). Mean differences with P-values $<0.05$ were considered to be statistically significant.

\section{Results}

Transfection of MUC4-siRNA into SKOV3 cells decreases Her2 stability via a proteosomal pathway. According to Moorthy et al, Her2 protein stability can be maintained by MUC4 in ovarian cancer cells (19). We examined Her2 stability in SKOV3 cells transfected with control-siRNA or siRNA against MUC4 with cycloheximide, an inhibitor of protein translation. As shown in Fig. 1, the Her2 protein had decreased stability since it disappeared earlier when transfected with siRNA against MUC4 and cycloheximide as compared to the control-siRNA and cycloheximide. To determine whether Her2 degradation upon attenuation of MUC4 by siRNA transfection was proteosome-mediated, we adopted MG-132, a type of proteasome inhibitor. While the expression level of Her2 was decreased at $0.5 \mathrm{~h}$ in SKOV3 cells transfected with MUC4-siRNA, the expression level of Her2 was not decreased at over $4 \mathrm{~h}$ in SKOV3 cells transfected with control-siRNA and MG-132 (Fig. 1) suggesting that degradation of Her2 by the transfection of MUC4-siRNA is proteasome-dependent.

Transfection of MUC4-siRNA or auranofin treatment into SKOV3 cells downregulates Her2 leading to decreased phospho-Akt. Since Akt is a well-known downstream target 
A

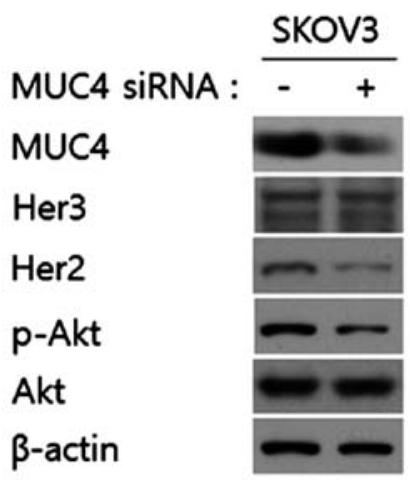

B

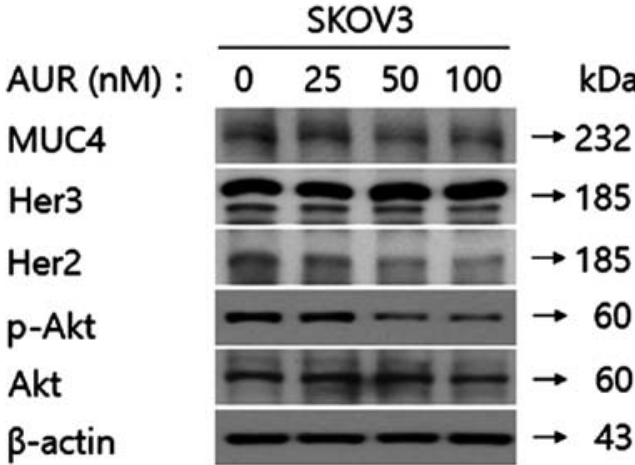

Figure 2. Her2 is identified as the common molecular target protein by siRNA transfection against MUC4 and auranofin treatment in SKOV3 cells. (A) Transfection of MUC4-siRNA into SKOV3 cells downregulates the expression level of Her2 leading to a decreased phospho-Akt expression level. SKOV3 cells $\left(1 \times 10^{5}\right)$ were seeded in $6-\mathrm{cm}$ dishes and incubated at $37^{\circ} \mathrm{C}$ in a humidified incubator containing $5 \% \mathrm{CO}_{2}$ in air for $18 \mathrm{~h}$. After incubation, the control or MUC4-siRNA was transfected. The cell lysates were immune-blotted with anti-MUC4, anti-Her3, anti-Her2, anti-phospho-Akt and anti-Akt antibodies. $\beta$-actin was used as the loading control. (B) Auranofin treatment in SKOV3 cells downregulates the expression level of Her2 leading to a decreased phosphoAkt expression level. SKOV3 cells $\left(1 \times 10^{5}\right)$ were seeded in 6 -cm dishes and incubated at $37^{\circ} \mathrm{C}$ in a humidified incubator containing $5 \% \mathrm{CO}_{2}$ in air for $18 \mathrm{~h}$. After incubation, the cells were treated with auranofin $(0,25,50$ or $100 \mathrm{nM})$. The cell lysates were immune-blotted with anti-MUC4, anti-Her3, anti-Her2, anti-phospho-Akt, and anti-Akt antibodies. $\beta$-actin was used as the loading control.

A

MUC4 siRNA : Cytoplasmic Her2 Nucleus FOXO3 Cytoplasmic B-actin Nucleus PARP1
B

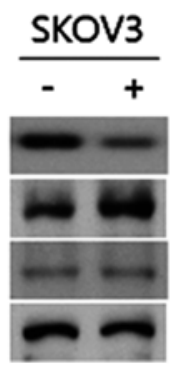

Cytoplasmic Her2

Nucleus FOXO3

Cytoplasmic B-actin Nucleus PARP1

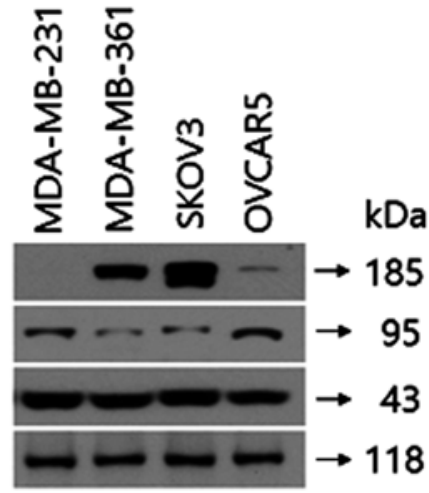

Figure 3. Expression level of nuclear FOXO3 is inversely correlated with the expression level of cytoplasmic Her2 in ovarian and breast cancer cell lines. (A) Transfection of MUC4-siRNA into SKOV3 cells downregulates the expression level of cytoplasmic Her2 leading to an increased expression level of nuclear FOXO3. SKOV3 cells $\left(1 \times 10^{5}\right)$ were seeded in $6-\mathrm{cm}$ dishes and incubated at $37^{\circ} \mathrm{C}$ in a humidified incubator containing $5 \% \mathrm{CO}_{2}$ in air for $18 \mathrm{~h}$. After incubation, the control or MUC4-siRNA was transfected. The cell lysates were immune-blotted using the cytosol/nuclear fractional buffer with anti-Her2 and anti-FOXO3 antibodies. $\beta$-actin was used as the cytoplasmic fraction loading control. PARP1 was used as the nuclear fraction loading control. (B) The expression level of nuclear FOXO3 was increased in ovarian and breast cancer cell lines with a low Her2 expression level. MDA-MB-231, MDA-MB-361, OVCAR5 and SKOV3 cells $\left(1 \times 10^{6}\right)$ were seeded in $10-\mathrm{cm}$ dishes and incubated at $37^{\circ} \mathrm{C}$ in a humidified incubator containing $5 \% \mathrm{CO}_{2}$ in air for $18 \mathrm{~h}$. After incubation, the cell lysates were immune-blotted using the cytosol/nuclear fractional buffer with anti-Her2 and anti-FOXO3 antibodies. $\beta$-actin was used as the cytoplasmic fraction loading control. PARP1 was used as the nuclear fraction loading control.

of Her2, we investigated whether the expression level of phospho-Akt was regulated by transfection of MUC4-siRNA in SKOV3 cells by carrying out western blot analysis. As shown in Fig. 2A, MUC4-siRNA transfection in SKOV3 cells decreased the expression level of phospho-Akt, but did not affect the expression level of total Akt. Recently, we reported that auranofin has anticancer activity via activation of FOXO3 in SKOV3 cells. In a previous study, we demonstrated that auranofin inhibited IKK $\beta$ leading to the promotion of FOXO3 translocation from the cytoplasm into the nucleus. Wenhui et al reported that IKK $\beta$ could be regulated by the PI3K/Akt/GSK3 $\beta$ pathway in colonic smooth muscle (22). Thus, we addressed whether auranofin treatment in SKOV3 cells affected MUC4, phospho-Akt and Akt expression levels. As shown in Fig. 2B, auranofin treatment in SKOV3 cells decreased the level of phospho-Akt, but did not alter the expression levels of MUC4 or Akt. Notably, transfection of MUC4-siRNA or auranofin treatment did not downregulate the protein expression level of Her3. These results demonstrated that both MUC4-siRNA transfection and auranofin treatment in SKOV3 cells not only regulated Her 2 specifically but downstream signaling targets such as phospho-Akt as well.

Attenuation of MUC4 combined with auranofin treatment in SKOV3 cells synergistically activates FOXO3 translocation from the cytoplasm to the nucleus through the regulation of the Her2/Akt/FOXO3 pathway. FOXO3 is a tumorsuppressive transcriptional factor and known to be inactivated through translocation from the nucleus into the cytoplasm. To date, 3 representative kinases, Akt, Erk and IKK $\beta$ have been identified to induce this translocation of FOXO3 by phosphorylation (23). We examined the expression level of 


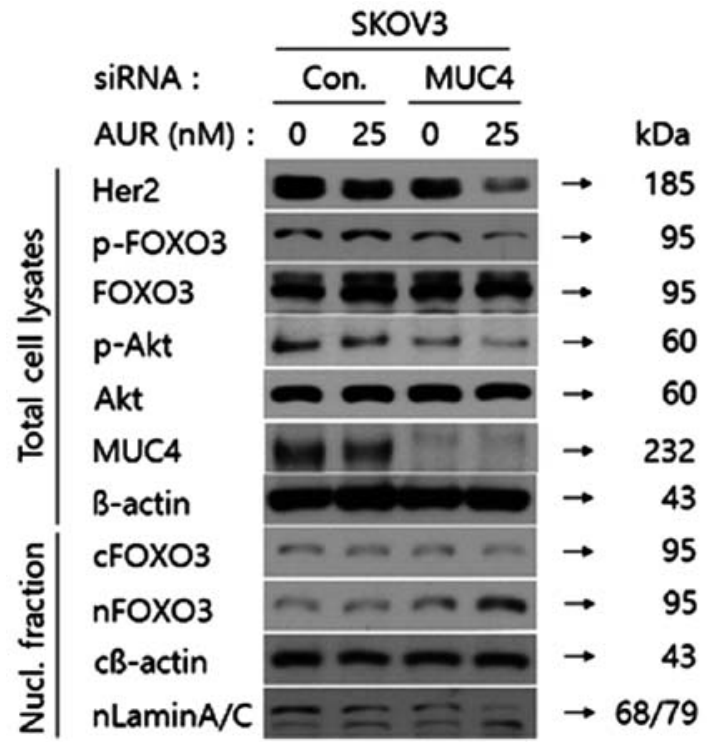

Figure 4. Combination of MUC4 by siRNA transfection with auranofin treatment in SKOV3 cell promotes FOXO3 translocation from the cytoplasm to the nucleus through regulation of the Her2/Akt/FOXO3 pathway. SKOV3 cells $\left(1 \times 10^{5}\right)$ were seeded in $6-\mathrm{cm}$ dishes and incubated at $37^{\circ} \mathrm{C}$ in a humidified incubator containing $5 \% \mathrm{CO}_{2}$ in air for $18 \mathrm{~h}$. After incubation, the control or MUC4-siRNA was transfected followed by the addition of auranofin ( 0 or $25 \mathrm{nM}$ ) into each dish for $24 \mathrm{~h}$. The cell lysates were immune-blotted with anti-MUC4, anti-Her2, anti-phospho-FOXO3, anti-FOXO3, anti-phosphoAkt and anti-Akt antibodies. $\beta$-actin was used as the loading control. In addition, the same cell lysates were immune-blotted using the cytosol/ nuclear fractional buffer with anti-FOXO3 antibodies. $\beta$-actin was used as the cytoplasmic fraction loading control. LaminA/C was used as the nuclear fraction loading control.

FOXO3 in both the cytoplasm and the nucleus of SKOV3 cells transfected with the control or MUC4-siRNA. As shown in Fig. 3A, the expression level of Her2 of the cytoplasmic fraction in SKOV3 cells transfected with MUC4-siRNA was downregulated compared to the control-siRNA. However, under the same conditions, the expression level of FOXO3 of the nuclear fraction (the active FOXO3) was upregulated. Then, in order to confirm the expression patterns of cytoplasmic Her2 and nuclear FOXO3 expression levels, we examined the cytoplasmic Her2 expression and the nuclear FOXO3 in the other cell lines with Her2 high (MDA-MB361 and SKOV3) and low expression (MDA-MB-231 and OVCAR5) levels. As shown in Fig. 3B, in MDA-MB-361 and SKOV3 cells the cytoplasmic the expression level of Her2 was markedly increased while in MDA-MB-231 and OVCAR5 cells the nuclear FOXO3 expression was upregulated, which was consistent with the results of Fig. 3A. Thus, it appears that Her2 may be the common molecular target protein by siRNA transfection against MUC4 and auranofin treatment in SKOV3 cells that activates FOXO3. Therefore, we next assessed whether combination of MUC4 by siRNA transfection with auranofin treatment in SKOV3 cells synergistically promoted FOXO3 translocation from the cytoplasm to the nucleus. As shown in Fig. 4, Her2 and phospho-Akt expression levels were significantly decreased in total cell lysates from SKOV3 cells transfected with MUC4-siRNA followed by auranofin treatment. Furthermore, the phospho-FOXO3 by Akt was downregulated under the same conditions compared to the other conditions, too. However, we could not detect any difference in the expression level of FOXO3 under any conditions. Thus, we adopted nuclear fractional western blot analysis to assess the expression level of FOXO3 in SKOV3 cells. As shown in Fig. 4. the expression level of FOXO3 was decreased in the cytoplasmic fraction from SKOV3 cells transfected with MUC4-siRNA followed by auranofin treatment but increased in the nuclear fraction, which implied that FOXO3 was translocated from the cytoplasm into the nucleus through the combination of MUC4 by siRNA transfection and auranofin treatment. These results revealed that attenuation of MUC4 by siRNA transfection combined with auranofin treatment in SKOV3 cells synergistically activated FOXO3 translocation from the cytoplasm to the nucleus through the regulation of the Her2/Akt/FOXO3 pathway.

Attenuation of MUC4 potentiates the anticancer activity of auranofin in SKOV3 cells. Then, we examined whether the anticancer activity of auranofin was potentiated in SKOV3 cells after attenuating MUC4 by siRNA transfection. We transfected SKOV3 cells with control or MUC4-siRNA and treated cells with auranofin ( 0 or $25 \mathrm{nM})$, and then assessed the survival and growth rate of SKOV3 cells using the WST-1, cell counting, and colony formation assays. As shown in Fig. 5A, although the sole treatment of auranofin $(25 \mathrm{nM})$ had a weak inhibitory effect ( $20 \%$ compared to the DMSO control) on SKOV3 cell survival, the combination of MUC4siRNA transfection with auranofin treatment significantly enhanced the antitumor activity of auranofin. In addition, the time-dependent cell counting assay results demonstrated the synergistic potentiation of the anticancer activity of auranofin (Fig. 5B), which was confirmed by colony formation assay using the same transfection and treatment conditions in SKOV3 cells (Fig. 5C). After $72 \mathrm{~h}$ of incubation, the number of cells in SKOV3 cells transfected with MUC4-siRNA followed by auranofin treatment $(25 \mathrm{nM})$ was significantly lower ( 3 times) than that of sole MUC4-siRNA or auranofin treatment. Furthermore, the 14-day results from the colony formation assay revealed that combination of MUC4 by siRNA transfection and auranofin treatment significantly inhibited the colony-forming ability of SKOV3 cells. These results demonstrated that attenuation of MUC4 by siRNA transfection potentiated the antitumor activity of auranofin on cell survival and proliferation of SKOV3 cells.

Attenuation of MUC4 potentiates the pro-apoptotic activity of auranofin in SKOV3 cells. To examine whether the pro-apoptotic activity of auranofin was potentiated in SKOV3 cells after attenuation of MUC4 using MUC4-siRNA transfection or not, we performed a TUNEL assay, Annexin V staining analysis, and western blot analysis. As shown in Fig. 6A, sole auranofin treatment ( $25 \mathrm{nM}$ for $2 \mathrm{~h}$ ) did not induce the apoptotic nuclei but auranofin treatment after MUC4-siRNA transfection resulted in the increased apoptotic DNA degradation in SKOV3 cells, which was confirmed by Annexin V staining analysis (Fig. 6B). Auranofin treatment after MUC4-siRNA transfection induced significant Annexin V-positive cell populations (15.26\% of apoptotic and dead cells and $6.53 \%$ of apoptotic cells) compared to the sole treatment (5.64\% of apoptotic and dead cells and $1.69 \%$ of apoptotic cells) in SKOV3 cells. In addition, auranofin treatment after MUC4-siRNA transfection induced the cleavage 
A

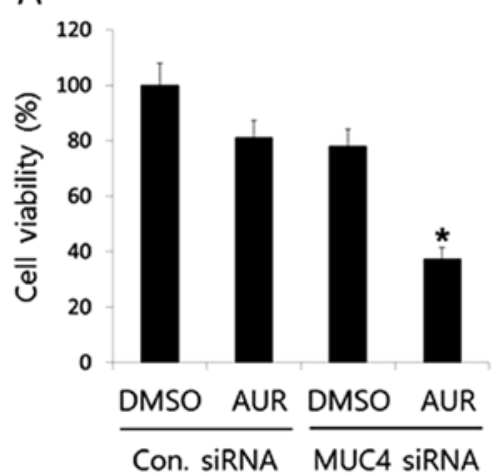

B

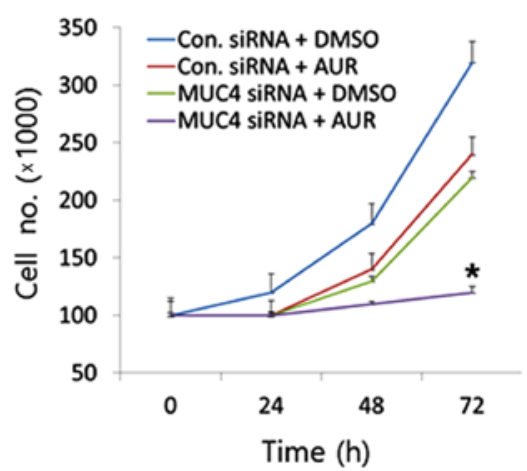

C

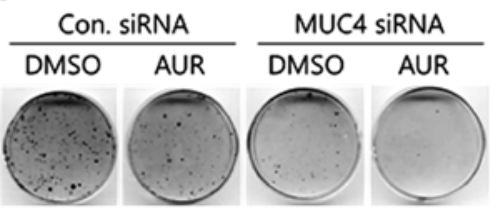

Figure 5. Anticancer effect from the combination of MUC4 by siRNA transfection and auranofin treatment in SKOV3 cells. (A) Cytotoxic effect of the combination of MUC4 by siRNA transfection with auranofin $(0$ or $25 \mathrm{nM})$ treatment in SKOV3 cells for $72 \mathrm{~h}$. SKOV3 cells $\left(1 \mathrm{x} 10^{3}\right)$ were seeded in a $96-$ well plate and incubated at $37^{\circ} \mathrm{C}$ in a humidified incubator containing $5 \% \mathrm{CO}_{2}$ in air for $18 \mathrm{~h}$. After incubation, the control or MUC4-siRNA was transfected followed by the addition of auranofin $(0$ or $25 \mathrm{nM})$ into each well for $48 \mathrm{~h}$. The cell viability was determined by the WST-1 assay and the relative cell survival rate percentage was calculated by dividing the optical density of each auranofin treatment by the optical density of the control (DMSO) treatment. (B) The cell growth inhibitory effect of the combination of MUC4 by siRNA transfection and auranofin (0 or $25 \mathrm{nM})$ treatment in SKOV3 cells for 0,24 , 48 and $72 \mathrm{~h}$. The number of cells were determined by hemocytometer. The significant P-values between the combination of MUC4 by siRNA transfection with auranofin treatment and the control group are indicated $\left({ }^{*} \mathrm{P}<0.05\right)$ calculated by paired t-test. This result is one of 3 replicates. The error bars represent the standard error. (C) Auranofin suppressed the colony-forming ability of SKOV3 cells. The number of colonies were determined by the colony formation assay. (A) SKOV3 cells (500 cells/plate) were treated with auranofin (100 nM) or the control (DMSO) for 7 days and stained with crystal violet solution. The representative images of the 3 assays are shown.

A

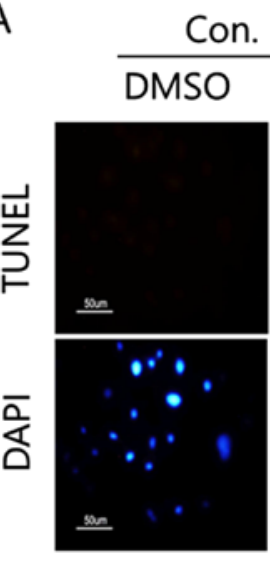

Con. SiRNA

AUR

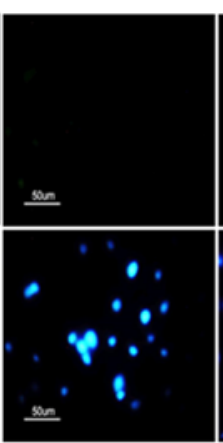

MUC4 SiRNA

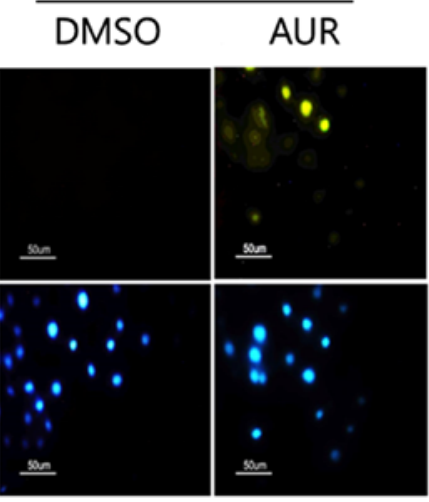

B

Con. SiRNA
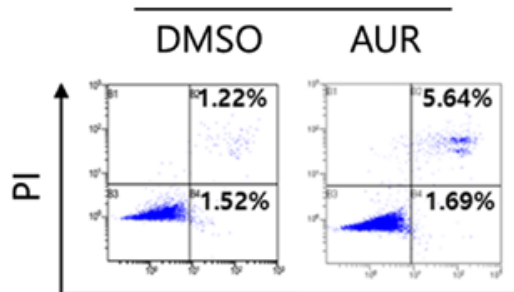

MUC4 SiRNA

\section{Annexin V}

Figure 6. Combination of MUC4 by siRNA transfection with auranofin treatment promotes cellular apoptosis in SKOV3 cells. (A) TUNEL assay. SKOV3 cells $\left(1 \times 10^{5}\right)$ were seeded in $6-\mathrm{cm}$ dishes and incubated at $37^{\circ} \mathrm{C}$ in a humidified incubator containing $5 \% \mathrm{CO}_{2}$ in air for $18 \mathrm{~h}$. After incubation, the control or MUC4-siRNA was transfected followed by the addition of auranofin ( 0 or $25 \mathrm{nM})$ into each dish for $2 \mathrm{~h}$. Then, the cells were fixed on the slides to determine the apoptosis by TUNEL assay. Nuclei were stained with DAPI (color-inverted to blue) and yellow cells were considered as apoptotic cells. Scale bar, $50 \mu \mathrm{m}$. (B) Annexin V staining assay. Under the same conditions as the TUNEL assay, SKOV3 cell were harvested, and subjected to Annexin V and propidium iodide staining to determine apoptosis. The representative images of the 3 assays are shown. 


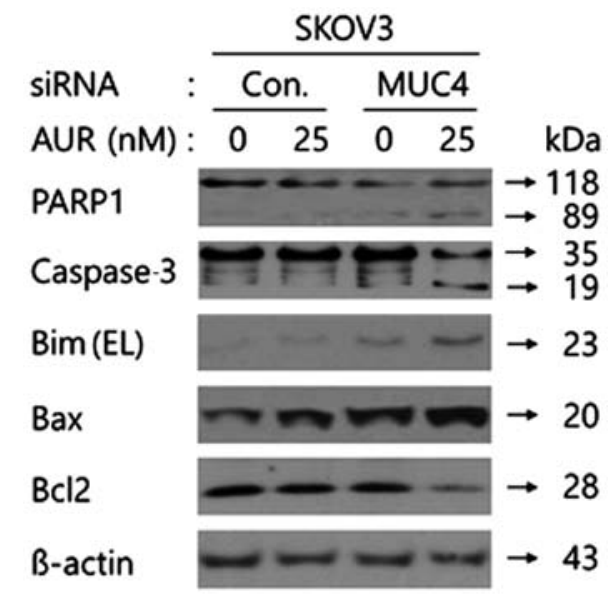

Figure 7. Combination of MUC4 by siRNA transfection with auranofin treatment potentiates the expression of apoptosis-related proteins in SKOV3 cells SKOV3 cells $\left(1 \times 10^{5}\right)$ were seeded in $6-\mathrm{cm}$ dishes and incubated at $37^{\circ} \mathrm{C}$ in a humidified incubator containing $5 \% \mathrm{CO}_{2}$ in air for $18 \mathrm{~h}$. After incubation, the control or MUC4-siRNA was transfected followed by the addition of auranofin ( 0 or $25 \mathrm{nM}$ ) into each dish for $48 \mathrm{~h}$. The cell lysates were immuneblotted with anti-PARP1, anti-caspase-3, anti-Bim, anti-Bax and anti-Bcl2 antibodies. $\beta$-actin was used as the loading control.

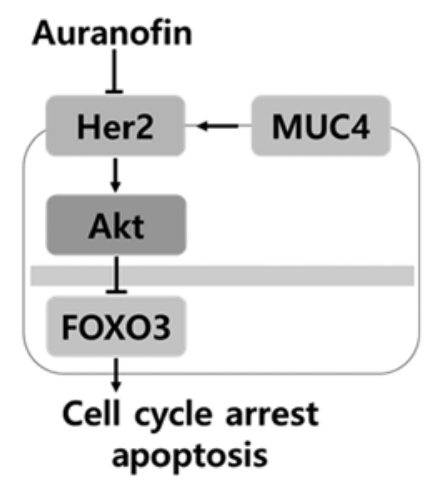

Figure 8. Schematic diagram showing the mechanism of the enhancement of the anticancer activity of auranofin by MUC4 attenuation through the Her2/Akt/FOXO3 pathway. MUC4 affected the Her2 protein stability and auranofin downregulated the Her2 protein expression level. Under the attenuation of MUC4 expression, auranofin promoted FOXO3 translocation from the cytoplasm into the nucleus to induce cell cycle arrest or pro-apoptotic proteins, as well as to downregulate the expression of an anti-apoptotic protein.

of PARP1 and caspase-3 and upregulated the expression of Bax and Bim EL in SKOV3 cells compared to the sole treatment, whereas auranofin treatment after MUC4-siRNA transfection decreased the $\mathrm{Bcl} 2$ expression level under the same conditions (Fig. 7). These results revealed that combination of MUC4 by siRNA transfection with auranofin treatment may exhibit its apoptotic effect through the caspase-3-mediated apoptosis mechanism in SKOV3 cells, the upregulation of the mitochondrial pro-apoptotic Bax and Bim proteins, and the downregulation of the anti-apoptotic $\mathrm{Bcl} 2$ protein expression.

\section{Discussion}

Ovarian cancer is one of the major causes of gynecological cancer-related deaths each year in the US. Although ovarian cancer after initial cytoreductive surgery has been generally shown to have a favorable response to combination chemotherapy (first line), advanced ovarian cancer is responsible for the worse prognosis of patients, due to acquired chemotherapy resistance $(24,25)$. While chemotherapies for ovarian cancer are currently being developed, the overall survival has not considerately increased since a significant number of these patients develop a resistance to the therapies and the majority of cancers susceptible to treatments in the beginning, become refractory (26). Therefore, a new targeted therapy needs to be developed for ovarian cancer resistance to chemotherapy.

MUC4 is a type of mucin glycoproteins and known as an activator of Her2 by inducing the dimerization of Her2 with other ErbB receptors $(27,28)$. There are lots of studies that have identified the existence of the MUC4/Her2 complex in various tumors and cancer cell lines (29,30). In addition, research has demonstrated that downregulation of MUC4 destabilizes HER 2 expression $(19,29)$. Similar to previous findings, the present study also revealed that silencing of MUC4 by siRNA transfection into SKOV3 cells decreases Her2 stability via a proteosomal pathway (Fig. 1). Notably, auranofin treatment in SKOV3 cells downregulated Her2 expression (Fig. 2B). Thus, both MUC4-siRNA transfection and auranofin treatment in SKOV3 cells regulate Her2.

Since Her2 is known to activate many downstream signaling targets including Akt (31), we investigated the effect of MUC4 knockdown by siRNA transfection in SKOV3 cells on Akt phosphorylation. As shown in Fig. 2B, we observed decreased Akt phosphorylation in MUC4 knockdown cells compared to control-siRNA transfected cells. Consistent with our present study, Kaur et al recently revealed that MUC4 was involved in the regulation of lipocalin2 through the Her2/Akt/NF- $\kappa \mathrm{B}$ signaling pathway in pancreatic cancer cells (6). Furthermore, auranofin treatment in SKOV3 cells induced decreased Akt phosphorylation. Therefore, we reasoned that the anticancer activity of auranofin may be enhanced by attenuating MUC4 expression through the regulation of the Her2/Akt pathway.

FOXO3 belongs to the human Forkhead-box (FOX) gene family which is characterized by a distinct Fork head DNA-binding domain (32). FOXO3 transcription factors exert functions in various processes including cellular differentiation, proliferation, cell cycle arrest, cell death, resistance to environmental stress and metabolism $(33,34)$. A great number of clinical studies have recently shown that the protein expression level of FOXO3 has far-reaching effects on cancer patient survival rates $(35,36)$. These observations revealed that FOXO3 could function as a prognostic marker in cancer, thereby FOXO3 regulation could be an anticancer therapeutic strategy. FOXO3 is known to be inactivated via a ubiquitin/proteasome system-mediated protein degradation after translocation from the nucleus into the cytoplasm. To date, 3 representative kinases such as Akt, Erk and IKK $\beta$ have been identified to induce this translocation of FOXO3 by phosphorylation (23).

Although we confirmed that the Her2 and phospho-Akt expression levels were significantly decreased in total cell lysates of SKOV3 cells transfected with MUC4-siRNA followed by auranofin treatment (Fig. 4), we could not detect any difference in the expression level of FOXO3. FOXO3 is a transcriptional factor and should be located in the nucleus to perform its transcriptional activity. Thus, researchers often try to perform the nuclear fractional western blotting 
by separating the cytoplasmic and nuclear protein fraction from cells to investigate the expression pattern of the specific transcriptional factor. Based on our recent studies on the activators for the FOXO3 transcriptional activity $(21,37)$, we adopted the nuclear fractional western blot analysis to assess the expression level of FOXO3 in SKOV3 cells transfected with MUC4-siRNA and auranofin treatment (Fig. 4) and observed that the expression level of FOXO3 was increased in the nucleus fraction implying the translocation of FOXO3 from the cytoplasm into the nucleus.

Recently, we reported that auranofin, a gold-combined drug used for rheumatoid arthritis in clinical treatment, has the anticancer activity in SKOV3 cells via regulation of the IKK $\beta /$ FOXO3 pathway (21). Including our previous studies, there have been many studies dealing with the anticancer activity of auranofin in various types of tumors (38-40). According to Li et al, auranofin exerted anticancer activity through the inhibition of the PI3K/AKT/mTOR signaling pathway in non-small cell lung cancer cells (41). Similarly, we observed auranofin-mediated downregulation of the phospho-Akt level in SKOV3 cells (Fig. 2B). Meanwhile, Tanaka et al investigated the cross-talk between IKK $\beta / \mathrm{NF}-\kappa \mathrm{B}$ and PDK1/Akt pathways and demonstrated that PDK1 activated NF- $\kappa \mathrm{B}$ signaling through IKK $\beta$ phosphorylation (42). PDK1 is known to activate (phosphorylate) Akt directly after being activated (phosphorylated) by PI3K. Thus, we surmised that there are two signaling pathways (Her2/ PI3K/PDK1/Akt or Her2/PI3K/PDK1/IKK $\beta$ axis) downstream of Her2. Currently, we are trying to examine whether auranofin treatment or MUC4 expression status in cancer cells affects PDK1 activation.

In order to develop a novel therapeutic strategy, we investigated the combinational effect of the attenuation of MUC4 by siRNA transfection and auranofin treatment in ovarian cancer cells. As shown in Fig. 5A and B, neither silencing of MUC4 by siRNA or auranofin treatment $(25 \mathrm{nM})$ suppressed the growth or viability of SKOV3 cells compared to the control siRNA or DMSO control. Colony formation assay results revealed that either silencing of MUC4 by siRNA or auranofin treatment $(25 \mathrm{nM})$ apparently decreased the colony formation ability of SKOV3 cells. We demonstrated that the synergistic anticancer activity of the combination was due to the downregulation of Her2 expression and phosphorylated Akt, which translocated FOXO3 from the cytosol into the nucleus and activated the transcriptional activity of FOXO3, inducing caspase-3-mediated apoptosis as well as Bim EL expression (Figs. 6 and 7). Recently, Lee et al revealed that treatment of entinostat, a class I histone deacetylase inhibitor, increased the anticancer activity of lapatinib, a Her2/EGFR dual tyrosine kinase inhibitor, in HER2-overexpressing breast cancer cells via FOXO3-mediated Bim expression (43). To the best of our knowledge, as described in Fig. 8, this is the first study demonstrating that the MUC4/Her2/Akt/FOXO3 signaling pathway is involved in regulating ovarian cancer cell development. Further studies analyzing tissue-micro array (TMA) from patients with ovarian cancer may be necessary to compare our current results to the clinical TMA data, which may provide the molecular basis of a novel anticancer therapeutic strategy. Collectively, auranofin regulated the Her2/Akt/FOXO3 signaling pathway in SKOV3 cells and may be used as a potential antitumor agent considering the expression of MUC4 in ovarian cancer patients.

\section{Acknowledgements}

The present study was supported by the Basic Science Research Program by the National Research Foundation of Korea (NRF) funded by the Ministry of Education, Science, and Technology (NRF-2014R1A6A3A04054307).

\section{References}

1. Hauber HP, Foley SC and Hamid Q: Mucin overproduction in chronic inflammatory lung disease. Can Respir J 13: 327-335, 2006.

2. Park HU, Kim JW, Kim GE, Bae HI, Crawley SC, Yang SC, Gum JR Jr, Batra SK, Rousseau K, Swallow DM, et al: Aberrant expression of MUC3 and MUC4 membrane-associated mucins and sialyl $\mathrm{Le}(\mathrm{x})$ antigen in pancreatic intraepithelial neoplasia. Pancreas 26: e48-e54, 2003.

3. Singh AP, Chauhan SC, Bafna S, Johansson SL, Smith LM, Moniaux N, Lin MF and Batra SK: Aberrant expression of transmembrane mucins, MUC1 and MUC4, in human prostate carcinomas. Prostate 66: 421-429, 2006

4. Voynow JA and Rubin BK: Mucins, mucus, and sputum. Chest 135: 505-512, 2009.

5. Rachagani S, Torres MP, Moniaux N and Batra SK: Current status of mucins in the diagnosis and therapy of cancer. Biofactors 35: 509-527, 2009.

6. Kaur S, Sharma N, Krishn SR, Lakshmanan I, Rachagani S, Baine MJ, Smith LM, Lele SM, Sasson AR, Guha S, et al: MUC4-mediated regulation of acute phase protein lipocalin 2 through HER2/AKT/NF- $\kappa \mathrm{B}$ signaling in pancreatic cancer. Clin Cancer Res 20: 688-700, 2014.

7. Chaturvedi P, Singh AP, Moniaux N, Senapati S, Chakraborty S, Meza JL and Batra SK: MUC4 mucin potentiates pancreatic tumor cell proliferation, survival, and invasive properties and interferes with its interaction to extracellular matrix proteins. Mol Cancer Res 5: 309-320, 2007.

8. Bafna S, Kaur S and Batra SK: Membrane-bound mucins: The mechanistic basis for alterations in the growth and survival of cancer cells. Oncogene 29: 2893-2904, 2010.

9. Inata J, Hattori N, Yokoyama A, Ohshimo S, Doi M, Ishikawa N, Hamada $\mathrm{H}$ and Kohno N: Circulating KL-6/MUC1 mucin carrying sialyl Lewis ${ }^{a}$ oligosaccharide is an independent prognostic factor in patients with lung adenocarcinoma. Int $\mathbf{J}$ Cancer 120: 2643-2649, 2007.

10. Schroeder JA, Masri AA, Adriance MC, Tessier JC, Kotlarczyk KL, Thompson MC and Gendler SJ: MUC1 overexpression results in mammary gland tumorigenesis and prolonged alveolar differentiation. Oncogene 23: 5739-5747, 2004.

11. Abd Elazeez TA, El-Balshy A-L, Khalil MM, El-Tabye MM and Abdul-Halim H: Prognostic significance of P27 (Kip 1) and MUC1 in papillary transitional cell carcinoma of the urinary bladder. Urol Ann 3: 8-13, 2011.

12. Patriarca C, Colombo P, Pio Taronna A, Wesseling J, Franchi G, Guddo F, Naspro R, Macchi RM, Giunta P, Di Pasquale M, et al: Cell discohesion and multifocality of carcinoma in situ of the bladder: New insight from the adhesion molecule profile (e-cadherin, Ep-CAM, and MUC1). Int J Surg Pathol 17: 99-106, 2009.

13. Du L, Qian X, Dai C, Wang L, Huang D, Wang S and Shen X: Screening the molecular targets of ovarian cancer based on bioinformatics analysis. Tumori 101: 384-389, 2015.

14. Slichenmyer WJ and Fry DW: Anticancer therapy targeting the erbB family of receptor tyrosine kinases. Semin Oncol 28 (Suppl 16): S67-S79, 2001.

15. Schmidt M, Lewark B, Kohlschmidt N, Glawatz C, Steiner E, Tanner B, Pilch H, Weikel W, Kölbl H and Lehr HA: Long-term prognostic significance of HER-2/neu in untreated node-negative breast cancer depends on the method of testing. Breast Cancer Res 7: R256-R266, 2005.

16. Ross JS and Fletcher JA: The HER-2/neu oncogene in breast cancer: Prognostic factor, predictive factor, and target for therapy. Oncologist 3: 237-252, 1998. 
17. Ladjemi MZ, Jacot W, Chardès T, Pèlegrin A and NavarroTeulon I: Anti-HER2 vaccines: New prospects for breast cancer therapy. Cancer Immunol Immunother 59: 1295-1312, 2010.

18. Hynes NE and Stern DF: The biology of erbB-2/neu/HER-2 and its role in cancer. Biochim Biophys Acta 1198: 165-184, 1994.

19. Ponnusamy MP, Seshacharyulu P, Vaz A, Dey P and Batra SK: MUC4 stabilizes HER2 expression and maintains the cancer stem cell population in ovarian cancer cells. J Ovarian Res 4: 7, 2011.

20. Ponnusamy MP, Singh AP, Jain M, Chakraborty S, Moniaux N and Batra SK: MUC4 activates HER2 signalling and enhances the motility of human ovarian cancer cells. Br J Cancer 99: 520-526, 2008.

21. Park SH, Lee JH, Berek JS and Hu MC: Auranofin displays anticancer activity against ovarian cancer cells through $\mathrm{FOXO} 3$ activation independent of p53. Int J Oncol 45: 1691-1698, 2014.

22. Hu W, Li F, Mahavadi S and Murthy KS: Upregulation of RGS4 expression by IL-1beta in colonic smooth muscle is enhanced by ERK1/2 and p38 MAPK and inhibited by the PI3K/Akt/GSK3beta pathway. Am J Physiol Cell Physiol 296: C1310-C1320, 2009.

23. Fu Z and Tindall DJ: FOXOs, cancer and regulation of apoptosis. Oncogene 27: 2312-2319, 2008.

24. Siegel R, Naishadham D and Jemal A: Cancer statistics, 2012. CA Cancer J Clin 62: 10-29, 2012.

25. Banerjee $S$ and Kaye SB: New strategies in the treatment of ovarian cancer: Current clinical perspectives and future potential. Clin Cancer Res 19: 961-968, 2013.

26. Shigetomi H, Higashiura $\mathrm{Y}$, Kajihara $\mathrm{H}$ and Kobayashi $\mathrm{H}$ Targeted molecular therapies for ovarian cancer: An update and future perspectives (Review). Oncol Rep 28: 395-408, 2012.

27. Singh AP, Moniaux N, Chauhan SC, Meza JL and Batra SK: Inhibition of $M U C 4$ expression suppresses pancreatic tumor cell growth and metastasis. Cancer Res 64: 622-630, 2004.

28. Carraway KL, Perez A, Idris N, Jepson S, Arango M, Komatsu M, Haq B, Price-Schiavi SA, Zhang J and Carraway CA: Muc4/sialomucin complex, the intramembrane ErbB2 ligand, in cancer and epithelia: To protect and to survive. Prog Nucleic Acid Res Mol Biol 71: 149-185, 2002.

29. Chaturvedi P, Singh AP, Chakraborty S, Chauhan SC, Bafna S, Meza JL, Singh PK, Hollingsworth MA, Mehta PP and Batra SK: MUC4 mucin interacts with and stabilizes the HER2 oncoprotein in human pancreatic cancer cells. Cancer Res 68: 2065-2070, 2008.

30. Ramsauer VP, Pino V, Farooq A, Carothers Carraway CA, Salas PJ and Carraway KL: Muc4-ErbB2 complex formation and signaling in polarized CACO-2 epithelial cells indicate that Muc4 acts as an unorthodox ligand for ErbB2. Mol Biol Cell 17: 2931-2941, 2006.
31. Holbro T and Hynes NE: ErbB receptors: Directing key signaling networks throughout life. Annu Rev Pharmacol Toxicol 44: 195-217, 2004.

32. Katoh $\mathbf{M}$ and Katoh M: Human FOX gene family (Review). Int $\mathbf{J}$ Oncol 25: 1495-1500, 2004

33. Alvarez B, Martínez-A C, Burgering BM and Carrera AC: Forkhead transcription factors contribute to execution of the mitotic programme in mammals. Nature 413: 744-747, 2001.

34. Furukawa-Hibi Y, Kobayashi Y, Chen C and Motoyama N: FOXO transcription factors in cell-cycle regulation and the response to oxidative stress. Antioxid Redox Signal 7: 752-760, 2005.

35. Lu M,Zhao Y, Xu F, Wang Y, Xiang J and Chen D: The expression and prognosis of FOXO3a and Skp2 in human ovarian cancer. Med Oncol 29: 3409-3415, 2012.

36. Fei M, Zhao Y, Wang Y, Lu M, Cheng C, Huang X, Zhang D, $\mathrm{Lu} \mathrm{J}, \mathrm{He} \mathrm{S}$ and Shen A: Low expression of Foxo3a is associated with poor prognosis in ovarian cancer patients. Cancer Invest 27: 52-59, 2009.

37. Park SH, Chung YM, Ma J, Yang Q, Berek JS and Hu MC: Pharmacological activation of FOXO3 suppresses triple-negative breast cancer in vitro and in vivo. Oncotarget 7: 42110-42125, 2016.

38. Gandin V, Fernandes AP, Rigobello MP, Dani B, Sorrentino F, Tisato F, Björnstedt M, Bindoli A, Sturaro A, Rella R, et al: Cancer cell death induced by phosphine gold(I) compounds targeting thioredoxin reductase. Biochem Pharmacol 79: 90-101, 2010.

39. Schuh E, Pflüger C, Citta A, Folda A, Rigobello MP, Bindoli A, Casini A and Mohr F: Gold(I) carbene complexes causing thioredoxin 1 and thioredoxin 2 oxidation as potential anticancer agents. J Med Chem 55: 5518-5528, 2012.

40. Pessetto ZY, Weir SJ, Sethi G, Broward MA and Godwin AK: Drug repurposing for gastrointestinal stromal tumor. Mol Cancer Ther 12: 1299-1309, 2013.

41. Li H, Hu J, Wu S, Wang L, Cao X, Zhang X, Dai B, Cao M, Shao R, Zhang R, et al: Auranofin-mediated inhibition of $\mathrm{PI} 3 \mathrm{~K} / \mathrm{AKT} / \mathrm{mTOR}$ axis and anticancer activity in non-small cell lung cancer cells. Oncotarget 7: 3548-3558, 2016.

42. Tanaka H, Fujita $\mathrm{N}$ and Tsuruo T: 3-Phosphoinositide-dependent protein kinase-1-mediated IkappaB kinase beta (IkkB) phosphorylation activates NF-kappaB signaling. J Biol Chem 280: 40965-40973, 2005.

43. Lee J,Bartholomeusz C,MansourO,Humphries J,HortobagyiGN, Ordentlich P and Ueno NT: A class I histone deacetylase inhibitor, entinostat, enhances lapatinib efficacy in HER2-overexpressing breast cancer cells through FOXO3-mediated Bim1 expression. Breast Cancer Res Treat 146: 259-272, 2014. 